\title{
In-Situ Characterization of Individual Building Blocks for Nanophotonic Solar Cells by Correlative Microscopy
}

\author{
Sebastian W. Schmitt ${ }^{1,2}$, George Sarau ${ }^{1,2}$ and Silke H. Christiansen ${ }^{1,2,3}$ \\ ${ }^{1 .}$ Helmholtz-Zentrum Berlin für Materialien und Energie, Institute Nanoarchitectures for Energy \\ Conversion, Hahn-Meitner-Platz 1, 14109 Berlin / Germany. \\ 2. Max Planck Institute for the Science of Light, Photonic Nanostructures, Günther-Scharowsky-Str. 1, \\ 91058 Erlangen / Germany. \\ ${ }^{3 .}$ Freie Universität Berlin, Department of Physics, Kaiserswerther Str. 16-18, 14195 Berlin / Germany.
}

A sophisticated optoelectronic characterization of single photonic nanostructure properties is crucial for a fast and straightforward advancement in the emerging field of nanophotonics. Here, we show the insitu optoelectronic characterization of an individual inverted silicon nanocone (SiNC) based solar cell inside a scanning electron microscope (SEM) equipped with an electrical nanoprober, an electron beam induced current (EBIC) and photocurrent amplifier and an optical fiber coupled with a multi-mode laser source located outside the SEM chamber. We present the identification of a pn-junction within the SiNC device, as well as dark and illuminated I-V characteristics, which permit to draw conclusions on its photovoltaic power generation. In particular, the dependence of the SiNC power generation on the intensity and wavelength of the incident light will be shown. While the generated power rises with illumination intensity, the wavelength dependent power generation of the device is strongly related to photonic modes inside the SiNC. Using additional photoluminescence measurements, the modulation in photocurrent of the device can be identified with photonic modes in the individual SiNC.

The integration of $\mathrm{Si}$ nanophotonic building blocks into optoelectronic devices is tedious, and controllable workflows place fundamentally new demands on metrology. Optoelectronic properties of the device-integrated structures have to be probed at individual level, since the mode formation in photonic resonators is very sensitive to their environment. In particular, their optical properties can be modified by coatings and contacts, attached analytes (e.g. cells, molecules) when it comes to optical sensing, and the direct vicinity of other structures as it occurs in densely packed arrays. Furthermore, electrical characteristics of nanoscopic building blocks usually depend on their high surface to volume ratios, and accordingly vary with geometry.

To this end, the presented work introduces a new measurement setup, which permits the in-situ determination of the optoelectronic characteristics of individual nanophotonic building blocks i.e. the nanoscopic unit of a photonic solar cell. The setup consists of an SEM that is equipped with an electrical nanoprober and a multi-mode laser source, which is fiber coupled to the inside of the microscope chamber. Hereby, it accounts for dark and illuminated current-voltage (I-V) characteristics measurements of nanophotonic building blocks. Furthermore, it permits EBIC measurements, to identify charge separating interfaces, such as e.g. a pn-junction, within nanoscopic devices (Fig. 1 a, b) [1]. For an initial demonstration of the feasibility and importance of the present setup to be applied for all sorts of nano-architecture optoelectronics, we selected a solar cell which consists of a single inverted SiNC [2,3]. We present the identification of a pn-junction within the SiNC device (Fig. $1 \mathrm{c}$ ), as well as dark and illuminated I-V characteristics which permit to draw conclusions on its photovoltaic power generation (Fig. 2 a). In particular, the dependence of the SiNC power generation on the intensity and wavelength of the incident light will be shown. While the generated power rises with illumination 
intensity, the wavelength dependent power generation of the device is related to photonic modes inside the SiNC. Using additional photoluminescence (PL) measurements the modulation in the device photocurrent can be identified with modes in the individual SiNC (Fig. 2 b).

\section{References:}

[1] SW Schmitt et al, Nanoscale 6 (2014), p. 7897.

[2] SW Schmitt et al, Sci. Rep. 5 (2015), p. 17089.

[3] G Shalev et al, Sci. Rep. 5 (2015), p. 8570.

a)

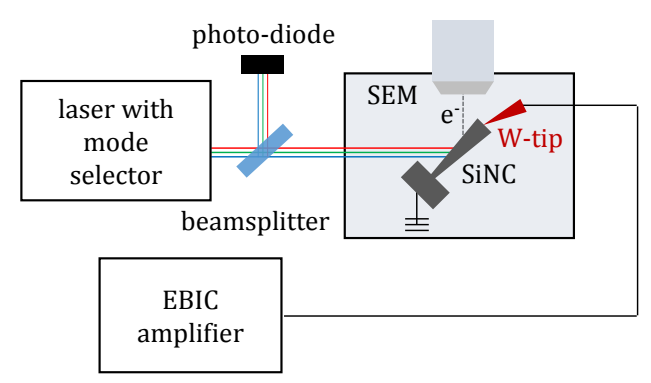

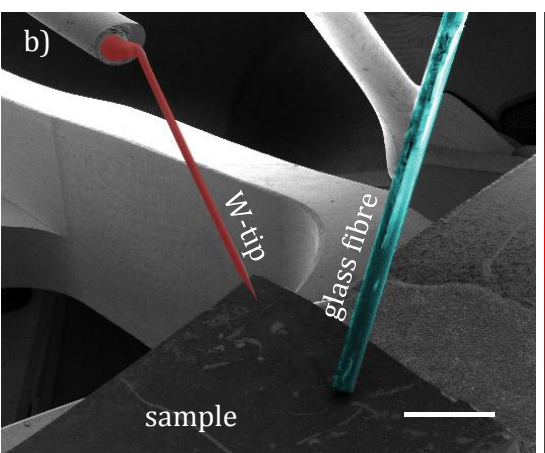

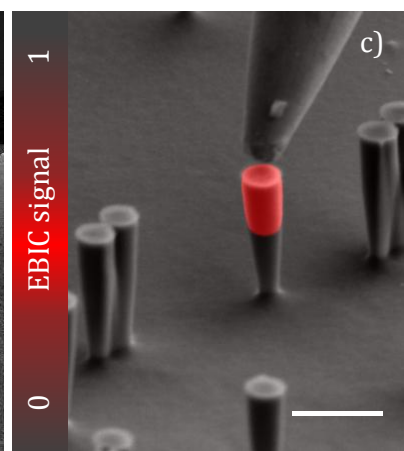

Figure 1. a) Schematic of the measurement setup. b) Overview of the experimental setup inside the SEM chamber showing the sample, the W-tip for contacting individual nano-structures and the multimode fiber for laser light illumination. Fiber and W-tip are mounted on nano-manipulators for maneuvering to the point of measurement (scale bar: $1 \mathrm{~mm}$ ). c) SEM image of an individually contacted SiNC ( $45^{\circ}$ tilt view). The EBIC mapping is superimposed in red color. EBIC signal intensities are normalized so that the scale shows values between 0 and 1 (scale bar: $2 \mu \mathrm{m}$ ).
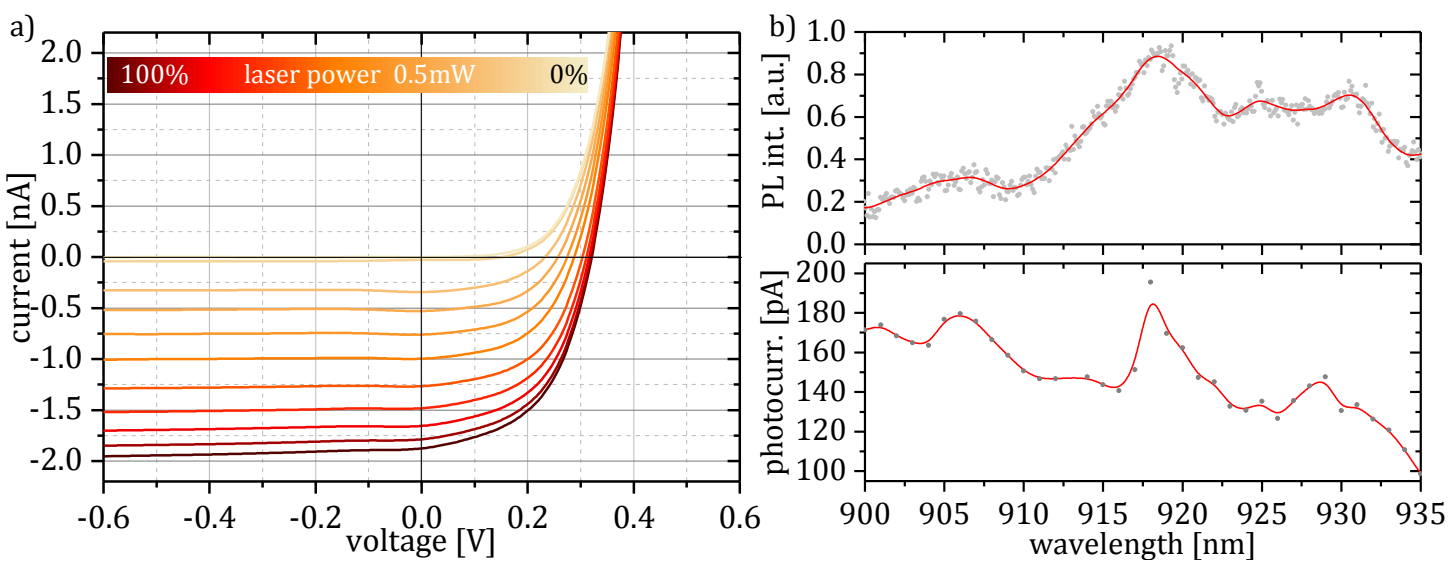

Figure 2. a) I-V characteristics of the individual SiNC under illumination with 900nm laser light at varying intensities between 0 and $0.5 \mathrm{~mW}$ with steps of $0.05 \mathrm{~mW}$. b) upper panel: Normalized PL intensity distribution of the individual SiNC diode excited with a $660 \mathrm{~nm} \mathrm{cw}$ laser at $1.28 \mathrm{~mW}$. lower panel: Photocurrent emerging from the individual SiNC device for a wavelength variation between 900 and $935 \mathrm{~nm}$ and constant laser power $(0.1 \mathrm{~mW})$. 